\title{
L'introduction de l'ancien français dans l'université française (1870-
} 1900)

\section{Gabriel Bergounioux}

\section{Citer ce document / Cite this document :}

Bergounioux Gabriel. L'introduction de l'ancien français dans l'université française (1870-1900). In: Romania, tome 112 n445446, 1991. pp. 243-258;

doi : https://doi.org/10.3406/roma.1991.1670

https://www.persee.fr/doc/roma_0035-8029_1991_num_112_445_1670

Fichier pdf généré le 07/04/2018 


\title{
MÉLANGES
}

\author{
L'INTRODUCTION \\ DE L'ANCIEN FRANÇAIS \\ DANS L'UNIVERSITÉ FRANÇAISE \\ (1870-1900)
}

\begin{abstract}
À s'en tenir aux affiches des facultés des lettres, dont lc dépôt aux Archives Nationales couvre les années 1845-1897 (de manière exhaustive à partir de 1875) ', l'enseignement de l'ancien français ², qui apparaît dans les vingt dernières années du $\mathrm{xIX}^{\mathrm{e}}$ siècle, semble perdre tout le prestige qui s`attache aux sciences pionnières. Partout, la réglementation des examens - licence et agrégation - et le prétexte de la littérature autorisent seuls l'introduction de cours dans les facultés de lettres. L'influence des jeunes romanistes (P. Meyer et G. Paris notamment) auprès des responsables de la Direction de l'Enseignement Supérieur du Ministère de l'Instruction Publique fut déterminante : l'administration imposait une discipline qui ne parvenait pas à s'implanter d'elle-même dans l'Université.
\end{abstract}

1. Cf. G. Bergounioux (1990). Voir note 24, infra.

2. On utilisera désormais la notation AF pour sténographier le syntagme ancien français. 
L'AF dans l'université contre les universitaires :

Si l'AF s'est imposé, c'est contre la définition et l'image traditionnelle des facultés de lettres, et contre la volonté des enseignants en poste. Création voulue par des maîtres qui appartenaient au Collège de France (M. Bréal, G. Paris, P. Meyer), à la IV section de l'École Pratique des Hautes Études (M. Bréal et G. Paris) et à l'École des Chartes (P. Meyer), soutenue par quelques hauts fonctionnaires, définie par des programmes décidés dans des cabinets ministériels, l'introduction de l'AF ne pouvait que détoner dans les cursus traditionnels des facultés. Encore avait-on pris soin que par son statut - des maîtrises de conférences -, cet enseignement parût subordonné aux chaires de littérature française occupées par une autre génération de professeurs.

L'apparition de l'AF correspond à l'émergence de l'université moderne ${ }^{3}$ : d'une certaine manière, elle en est un des symptômes et la conséquence. Il faut se rappeler que la création du corps des maîtres de conférences date de 1877 et que l'AF est une des premières disciplines à en bénéficier. En donnant un contenu scientifique aux épreuves de la licence et de l'agrégation, l'étude de l'histoire de la langue française remplissait une triple fonction : d'abord, objectiver les critères d'évaluation des futurs enseignants (privilégiant la compétence technique contrc le goût littéraire); ensuite, rapprocher l'enseignement universitaire du modèle allemand; enfin, rompre la continuité entre l'enseignement secondaire dominé par le latin et les cours de faculté. Cette conséquence est décisive puisqu'il devient pratiquement impossible de préparer la licence ou l'agrégation sans suivre des cours. Un double objectif était atteint : assurer la présence d'un certain auditoire dans les facultés et disqualifier des personnels exclusivement rompus aux pratiques des lycées (disons, le thème et la version en grec et en latin).

En retour, les romanistes semblent aussi avoir souhaité la reconnaissance de l'AF pour créer un précédent. À partir d'un domaine auquel peu de professeurs en place pouvaient s'opposer tant il semblait légitime (puisque doté d'une littérature spécifique

3. A. Prost : « Nous devons aux vingt années 1877-1896 la fondation de notre enseignement supérieur, nous leur devons son organisation scolaire et administrative, et jusqu’à ses murs ", p. 240. Voir note 24, infra. 
déjà étudiée en Allemagne, ce qui rendait urgent une réappropriation nationale), il devenait concevable d'introduire d'autres disciplines qui participeraient à la constitution d'un espace pour une science $d u$ langage. Tel apparaît l'enjeu : comment échapper aux sujétions littéraires ou historiques assumées par la philologie et faire admettre un enseignement plus proprement linguistique ${ }^{4}$ ?

L'AF occupait une place charnière dans cette ambition : impliquant le travail sur manuscrits, il nécessitait aussi une explication en termes de phonétique historique. S'il participait à la sacralisation académique de la langue française, il ne pouvait contourner la question des dialectes. Enfin, l'approche des problèmes revenait à structurer les différents domaines de la langue : la partition en phonétique, lexique, morpho-syntaxe et sémantique du Cours de grammaire historique de la langue française d'A. Darmesteter organisait une méthodologie dont le transfert pouvait être accompli sur n'importe quelle langue ou patois et servir de modèle à tous les enseignements de lettres autres que la littérature et l'histoire.

Ce caractère novateur dans les protocoles d'études se retrouve dans le fait que, parmi les enseignements déclarés de 1845 à 1897 , la linguistique historique (ou comparée, les deux termes semblent interchangeables) concerne pour l'essentiel trois domaines : le sanscrit, bien sûr, et, de manière indissociable, le provençal et l'AF ${ }^{5}$. Une des conséquences de cette présence de l'AF est qu'elle en viendra à justifier une certaine disymétrie dans l'intitulé des chaires. Toutes les chaires dévolues à une littérature autre que française (latine, grecque, anglaise, allemande, italienne, espagnole, arabe, sanscrite...) modifièrent leur appellation entre 1877 et 1885 afin de porter non plus « Littérature (latine, grecque, etc) " mais « Langue et littérature (latines, grecques, etc.) ». Seule la «Littérature française »

4. À la différence de l'enseignement dispensé dans les grandes écoles et les établissements spécialisés - Collège de France, Langues O., E.P.H.E. où l'absence de reconnaissance professionnelle des diplômes délivrés et la compréhension de langues exotiques ou disparues justifiaient per se une approche linguistique.

5. Le travail sur le celtique de Dottin, à Rennes, est plus historique ou ethnographique que grammatical ; quant à la conférence de Grammont, à Dijon, sur le lituanien, elle semble destinée à dissuader quelque improbable auditoire. 
demeura séparée de la "langue française » puisque aucune chaire, jusqu'à 1897 au moins, n'est désignée par : «Langue et littérature françaises $\gg 6$.

L'université s'en tient à une conception immanente de la langue française : elle ne peut en faire un thème d'étude qu'à s'en saisir comme objet d'élection dans un style, dans le registre du goût. Pour que le français soit apprćhendé scientifiquement, il fallait le détour par une diachronie qui restitue aux mots familiers une épaisseur, aux textes une opacité, à la langue un passé 7 . Les grands dictionnaires, les grammaires de référence jusqu'à 1870 n'ont pas été composés par des universitaires : le champ de ces travaux eût dérogé à des professeurs de faculté plus intéressés par le latin et le grec. La perspective d'une histoire, le déploiement multiséculaire du français rendait une légitimité à des ouvrages comme les grammaires de L. Clédat ou la participation d'A. Darmesteter au Dictionnaire Général.

Il ne s'agit pas d'instruire le procès des facultés de lettres de l'époque (avant 1877) dont l'inanité est avérée. Pour tout ce qui concerne l'AF, elles sont simplement représentatives d'un désintérêt plus général. Ce n'est qu'au début du xix ${ }^{\mathfrak{C}}$ que l'engouement pour le moyen-âge a atteint une certaine visibilité intellectuelle : quelle meilleure preuve en donner que l'absence même de désignation pour ce qu'il s'agissait d'étudier?

\section{L'enseignement de l'ancien français :}

La force de l'usage a pu faire oublier que l'idiome (ou les idiomes) parlé dans l'Ile-de-France -- et plus généralement dans les pays du nord de la Loire - entre le $\mathrm{vI}^{\mathrm{e}}$ et le $\mathrm{Xv}^{\mathrm{e}}$ siècles n'avait pas conservé de nom qui lui soit propre et qui permettrait de le distinguer de son avatar moderne. Que la convention d'une désignation comme "ancien français " est somme toute récente. Jusqu'à la fin du XIX siècle, il $\mathrm{y}$ a une hésitation entre plusieurs dénominations, sans

6. On écrira "Littérature française » (avec guillemets et majuscule) pour sténographier : chaire de littérature française. On sait que cette séparation s'est perpétuée jusqu'à aujourd'hui.

7. On pourrait suivre, comme Antoine Compagnon, l'effet en retour sur les études de littérature avec l'apparition du lansonisme. 
qu'aucunc ne semble avoir été dominante, et surtout pas celle de "gaulois", à la mode jusqu'au début du XIX" siècle, employée d'ailleurs parfois par confusion entre l'idiome celte et les origines du français ${ }^{8}$.

La justification universitaire de l'AF : Avec la redécouverte du moyen-âge par les historiens, notamment Augustin Thierry et de Sismondi, on a parlé de "roman", d' " ancienne langue française ". Il y a une recension des noms alors en usage qu'il reste à faire : nous avons restreint notre inventaire aux intitulés des cours. Non seulement, le comptage et le classement en sont facilités mais, au-delà du disparate des désignations, il est possible de saisir, dans ses effets institutionnels, une logique.

Pour qu'apparaisse de l'AF, il fallait que la littérature du moyen-âge figure dans les programmes. Ce n'est qu'à partir de 1880 que la définition du concours d'agrégation inclut une épreuve centrée sur un texte du moyen-âge, essentiellement La Chanson de Roland. Ce recours au décret (réglementation des recrutements d'enseignants) est sommairement justifié par une modification des programmes du second degré. En ajoutant à l'enseignement de la grammaire dans les lycées, à partir de 1875, quelques notions d'histoire de la langue, les fonctionnaires de l'Instruction Publique imposaicnt un enseignement qui durera après qu'une autre réforme du secondaire, en 1885, aura conduit au quasi-abandon de ces rudiments de grammaire historique. L'AF est donc requis par une définition des cours du second degré qui aura duré une dizaine d'années : au-delà, l'enseignement ne se soutient plus que de lui-même. S'il reste prévue une place dans les progammes des lycées pour la langue et la

8. C'est la thèse de l'origine gauloise du français, ravivée au début du siècle par l'Académie Celtique, devenue Société des Antiquaires de France, et encore soutenue, dans les années 1850 par Granier de Cassagnac et en 1869 par Boucherie : "M. Boucherie conclut et se résume en disant que l'ancienne langue gauloise devait avoir avec la langue latine les mêmes rapports que les patois français ont aujourd'hui avec les patois italiens. En un mot, la parenté qui unit actuellement ces deux idiomes, et qu'il suppose avoir été à peu près la même autrefois, scrait une parenté de sưur à sœur et non de fille à mère " dans Bull. de la S.E.L.R. (p. 27). 
littérature du moyen-âge, il semble que la pratique des établissements et des enseignants en fasse un vœu pieux.

On mesure aussi quelle dut être la résistance des professeurs de la Sorbonne en dépit des encouragements de quelques personnalités, comme le doyen Victor Le Clerc ${ }^{9}$. Pour qu'il puisse être question d'AF, il fallait que la dominante littéraire des études perpétuât le caractère subordonné de l'approche linguistique qui n'intervenait qu'à titre d'explication des textes.

Le cas Heinrich : Il existe bien une exception : Heinrich. D'origine allemande, Guillaume Heinrich est titulaire de la chaire de littérature étrangère de Lyon depuis 1863. Afin d'obtenir une promotion à Paris, où l'on annonce la création d'une chaire d'AF, il a recours aux travaux de la romanistique allemande pour tenter de devancer ses concurrents et prendre date.

Comme il est question d'implanter à la Sorbonne, à côté de la chaire d'AF, une chaire de grammaire comparée (sanscrit), Heinrich se prépare à postuler pour les deux en adjoignant à son enseignement en chaire une «conférence de grammaire comparée » dans laquelle il annonce :

en 1875-1876: "Classification des langues aryennes. Étude des racines, de l'étymologie, de la dérivation. Phonétique des langues classiques et des langues romanes. Formation de la vieille langue française $10 »$.

en 1876-1877 : «Grammaire générale des langues romanes considérées dans leurs rapports avec le latin. Révision, au point de vue des méthodes philologiques modernes, de la grammaire française". Las! Ce n'est pas une chaire mais une maîtrise de conférences qui est créée à Paris en 1877 et c'est Arsène Darmesteter qui en bénéficie. Il ne reste à Heinrich qu'à espérer la chaire de sanscrit :

9. Titulaire de la chaire d'Éloquence latine et doyen jusqu'à 1865, Le Clerc avait été chargé, par l'Académie des Inscriptions et Belles-Lettres, d'éditer une collection de textes de la littérature médiévale; enthousiasmé par ses découvertes, il se fit le prosélyte du médiévisme français à l'intérieur de l'Université.

10. On écrira en les soulignant les intitulés portés dans les cours. 
1877-1878 ; «Classification des langues de l'Europe. Principes généraux de l'étymologie, de la dérivation. Étude générale des langues germaniques $11 \gg$.

Deux fois hélas : en 1878, la maîtrise de conférences de "Langue et littérature sanscrites » est confiée à Bergaigne.

Heinrich, devenu doyen de la faculté des lettres de Lyon en 1875 , ne s'occupera plus jamais de linguistique ni de philologie.

Clédat, Darmesteter, Joret et les autres: Un an après la première conférence de Heinrich, un " cours complémentaire » de "Langue et littérature françaises du moyen-âge " est confié à Léon Clédat (Lyon, 1876) 12 : «M. Clédat, archiviste-paléographe, membre de l'École Française de Rome, chargé du cours, traitera de la littérature provençale et spécialement des poésies de Bertrand de Born" " Parallèlement, il consacre une partie de son enseignement à l'« Étude de la grammaire de la vieille langue française", reprenant la désignation proposée par Heinrich pour la première et la dernière fois. Les années suivantes, Clédat préférera parler de français tout court ou d'anciens textes de la langue française (Lyon, 1877-96). On pourrait rapprocher cette décision, tout à fait originale, du travail accompli en histoire du français et en dialectologie du lyonnais : comme si, pour Clédat, aucune séparation tranchée ne pouvait être établie, que ce soit dans l'espace (dialectal) ou dans la durée. Seules des contraintes éditoriales le feront revenir, pour un titre d'ouvrage, à l'intitulé de Heinrich ${ }^{13}$.

Un an après Lyon, le Ministère de l'Instruction Publique crée une maîtrise de conférences à Paris sous l'intitulé « Langue et littérature françaises du moyen-âge " (le cours complémentaire de Clédat à Lyon est promu au même statut avec le même intitulé). Grâce à Gaston Paris et Paul Meyer, Arsène Darmesteter est nommé à la

11. Nous avons montré (G. Bergounioux, à paraitre) en quoi des termes comme «étymologie », "phonétique » et "philologie» fonctionnaient moins comme des indices de la diffusion scientifique que comme des marqueurs auto-décernés de modernité dans le champ de la linguistique.

12. On fera suivre le nom de l'enseignant de la ville de son université et de la date à laquelle le cours est mentionné (1876 correspond à l'année universitaire 1876-1877).

13. Cf. Clédat. Voir note 24 , infra. 
Sorbonne en dépit des prétentions de Heinrich et de Joret (Aix-enProvence, 1876-96). Celui-ci, chargé de cours dans la chaire de "Littérature étrangère" avait annoncé, dès sa première année d'enseignement, une : "Conférence de philologie romane avec explications d'anciens textes français, provençaux, italiens et espagnols ».

À la différence de Hcinrich, il reprendra les années suivantes cet enseignement.

À partir de 1883, les maîtrises de conférences seront transformées en chaire de plein exercice : A. Darmesteter est le premier à être promu professeur de « Littérature française du moyen-âge et histoire de la langue française" (Paris, 1883). Le titre retenu, différent de celui de la maîtrise de conférences, est significatif à un double titre. D'abord, il opère une distinction nette entre ce qui relève de la littérature et ce qui relève de la langue, impliquant une certaine reconnaissance du recours à la phonétique ${ }^{14}$. Ensuite, il évite, par sa formulation, de prendre parti dans le différend qui oppose les romanistes parisiens à leurs homologues montpelliérains concernant le statut de la langue d'oc. On peut accorder que la lyrique méridionale appartient à la "littérature française du moyen-âge » sans réduire le provençal à un épisode de «l'histoire de la langue française ". À l'inverse, Clédat, promu professeur en 1885, conserve l'intitulé de sa maîtrise de conférences.

À qui revient alors le mérite d'avoir, le premier, dans les facultés, donné à son enseignement l'intitulé «ancien français»?

\section{Le nom d' " ancien français ":}

Joret (Aix, 1880) a introduit la formule en formant le nom composé ancien-français (avec un trait d'union). Voici ce que porte l'affiche annonçant son cours : «Histoire de la poésie épique et lyrique au moyen-âge, principalement en Allemagne et en Italie; étude comparée de la langue et de la littérature allemandes et

14. Sur la forclusion de la phonétique à l'intérieur du champ de la linguistique, of. G. Bergounioux (1992). Voir note 24, infra. 
anglaises ${ }^{15}$; grammaire comparée de l'ancien-français et du provençal et explication de La Chanson de Roland".

Alors qu'à partir de 1880 les principales facultés de lettres se doivent de déclarer un enseignement d'AF si elles veulent que leurs étudiants puissent bénéficier de bourses de licence et d'agrégation en justifiant d'une préparation conforme aux programmes, les dénominations sont extrêmement variables. Joret n'avait pas un prestige suffisant pour que d'autres enseignants l'imitent et la pluralité des désignations témoigne du caractère encore mal structuré du champ des études universitaires en AF. Voici les termes retenus :

- langue française du XII' siècle pour Benoist (Nancy, 1880);

- langue d'oül puis grammaire du vieux français pour Boucherie (Montpellier, 1879 et 1880);

la langue française au moyen-âge pour Étienne (Nancy, 1883-95) : au-delà, ancien français:

- vieux français pour Thomas (Toulouse, 1882) qui se rallie à ancien français à partir de 1888, et pour Loth (Rennes, 1884-1886).

À partir de 1883, on constate une unification autour d' " ancien français " qui tend à devenir exclusif dans les enseignements de Bourciez (Bordeaux, 1883), Darmesteter (Paris, 1886), Thomas (Paris, 1889-94) et Brunot (Paris, 1896), Joret (Aix, 1880) et Constans (Aix, 1888-96), Thomas (Toulouse, 1888) et Jeanroy (Toulouse, 1889-96), Ducros (Poitiers, 1889-90) et Étienne (Nancy, 1895).

Ne demeurent en marge de l'unification que Rennes - où Dottin (Rennes, 1892-96) prend la suite des cours de Loth (Rennes, 1884-85) en conservant vieux-français ${ }^{16}$ et deux villes où l'AF n'est pas étudié pour lui-même mais dans le cadre d'une comparaison dialectale : Lille et Montpellier.

\section{Les usages de l'ancien français :}

S'il y a eu un enseignement de l'AF dans les facultés de lettres, il en faut chercher le principe dans la politique de réforme universitaire

15. Depuis sa nomination à Aix en 1877 , Joret enseigne dans la chaire de Littérature étrangère.

16. Par exemple, en 1892 : «Phonétique du vieux-français ». 
et l'application dans la création des bourses de licence et d'agrégation. L'AF ne constitue pas un objet d'étude en tant que tel mais seulement une introduction aux épreuves de littérature du moyenâge des examens et concours. Il n'existe qu'un seul cours consacré à "L'origine du français : les dialectes " par Roy (Dijon, 1895), encore est-il sans lendemain.

Pourtant, d'autres cours appréhendèrent la question de l'AF à partir d'une autre dimension : quel est le statut de l'AF en regard du picard ou de l'occitan?

À Lille, Langlois est appelé dans une chaire de "Langue et littérature picarde et wallonne " à partir de $1888^{17}$. L'AF apparaissant comme un terme générique, Langlois hésite, dans les annonces de ses cours entre deux oppositions :

- "langue d'oül » (qui sous-entend «langue d'oc»), par exemple en 1891 : «Histoire de la littérature en langue d'oïl depuis ses origines jusqu'à la Renaissance ".

- "français vs picard", dès la première année (1888) et passim : «Grammaire comparee des dialectes wallons, picards et français ».

On voit qu'il manque déjà un terme qui permettrait de désambiguïser les deux acceptions : "français = langue d'oill " et "français $=$ un dialecte d'oïl parmi d'autres ". Il existait un vide structural que devait combler, dans les années qui suivirent, "francien».

L'AF et l'occitan : En-dehors de Lille, il n'existe aucun enseignement concernant les dialectes d'oïl. C'est donc par opposition à l'occitan qu'il est question d'AF. Mais l'occitan lui-même n'a pas de désignation uniforme : Clédat (Lyon, 1875-97) parle de provençal, adoptant le nom conventionnel popularisé par Mistral. Luchaire, après avoir utilisé la forme ancien-provençal ${ }^{18}$ (Bordeaux, 1877),

17. Le pouvoir central, si indifférent, voire hostile, aux patois dans l'enseignement primaire, encouragea au contraire la création de cours ou de chaires de langues régionales à Montpellier (1879), Toulouse (1881), Rennes (1883), Lyon (1885), Lille (1888), Aix (1891) et Bordeaux (1893).

18. Il est le seul à préciser "ancien", tous les autres usages s'en tiennent au seul "provençal». Il est vrai que Luchaire poursuivra sa carrière en histoire. 
propose langue d'oc (Bordeaux, 1878), désignation reprise par Chabaneau (Montpellier, 1879-88) et Thomas (Toulouse, 1882).

Pourtant, alors qu' "ancien français » va se généralisant, l'hésitation dure puisque Thomas revient à la forme provençal (Toulouse, 1883 et 1887) qui s'impose à Chabaneau (Montpellier, 1889-93), Bourciez (Bordeaux, 1890-91) et Constans (Aix, 1895-96), tandis que Clédat l'a toujours conservée à Lyon. Ce retour à la désignation d'un ensemble de dialectes par l'un d'eux pose un problème comparable à celui que rencontrait Langlois. Comme la domination du français pouvait justifier la projection dans le passé de "langue d'oil = français ", les succès du Félibrige déterminaient l'acception "provençal = langue d'oc $"$.

Il n'en est que plus significatif de voir Chabaneau (Montpellier, 1895-96) revenir à la forme langue d'oc, en opposition explicite avec langue d'oül. À ceci, deux raisons : premièrement, le Félibrige est contesté par une dissidence limousine menée par l'abbé Joseph Roux qui trouve la graphie et les thèmes de l'école mistralienne trop provençalistes au sens restreint du terme; or, Chabaneau appartient lui-même aux marches du domaine occitan, sa langue maternelle étant le nontronnais, un des dialectes du limousin parlé en Périgord. Deuxièmement, les Montpelliérains de la Société pour l'Étude des Langues Romanes, dont Chabaneau est un des membres les plus éminents, ont engagé une polémique avec Gaston Paris sur le statut des dialectes. G. Paris (1893) tente d'imposer l'idée d'une aire gallo-romane dont le français serait la langue littéraire. Les occitanistes ont donc intérêt à rappeler que le français est (ou fut) un dialecte parmi d'autres et que l'unité d'une langue peut être constituée par la somme de ses dialectes sans s'identifier à aucun en particulier.

Il reste à mentionner le cas de Bourciez (Bordeaux, 1892-96) qui, afin de bénéficier d'une chaire d'initiative locale -- il s'agissait d'une fondation municipale --, modifia l'intitulé de son cours : "Phonétique française et provençale » (1892) pour y faire figurer "L'idiome gascon, étude historique et géographique des sons et des formes " (1893) et «L'idiome gascon de Bordeaux » (1894-95).

"Ancien français" : les raisons d'un choix : Dans le remplacement de «vieux français» par "ancien français", on peut lire aussi la défiance pour des métaphores biologiques. Le succès limité du 
darwinisme linguistique en France (représenté par Darmesteter, précocement disparu, et Hovelacque, dont la position de linguiste était marginale) ne favorisait pas la plus mal fondée des métaphores. En effet, le "vieux français" est le contraire de ce qu'on en dit puisqu'il s'agit au contraire d'un «jeune français». Tout au plus, peut-on le qualifier d' "ancien" en regard de l'état de langue actuel.

On pense aussi à des stratćgies de reconnaissance institutionnelle : l'idée de "vieux » est facilement associée à celles de "poussiéreux " ou de "dépassé », "ancien » apparaissant plutôt comme équivalant à "vénérable", ainsi dans " grec ancien ». Si ces raisons ont joué, il est inutile d'en exagérer la force : on parle de "vieil anglais » ou de «vieux haut allemand » sans que ni l'incongruïté, ni le mépris ne s'y remarquent.

L' "Ancien français » permettait d'imposer une désignation qui sanctionne la constitution d'un objet indépendamment des études historiques - ce que ne permettait pas la formulation de «langue française du moyen-âge " - tout en postulant une autonomie de cet état de langue par rapport au français moderne. Une des preuves de la consistance que prend alors le domaine est la façon dont s'opère l'unification des formulations des cours. Pendant qu'un terme -. "ancien français" -- devient la référence obligée, d'autres se périment -- comme l'intitulé vieux français de Dottin (Rennes, 1896) qui a dû sembler bien démodé dans ces années.

Que «ancien français » soit devenu pour nous le nom même de ce qu'il désigne ne saurait nous faire oublier qu'il a fallu d'abord que ce français-là soit pensé comme spécifique par rapport au français moderne, ensuite qu'il unifie tous les dialectes face au "provençal " (lequel unifiait tous les dialectes d'oc de manière assez factuelle), enfin que, quoique langue morte, il puisse se distinguer des études littéraires ou historiques, c'est-à-dire que soient constituées une grammaire, une phonétique et même une lexicographie ${ }^{19}$ qui lui soient propres.

Du fait de l'absence de chaire de «Langue française», la reconnaissance d'une singularité phonétique et grammaticale s'établissait, dans l'Université, sans référence à l'état moderne de la langue. Pour atteindre à une certaine ressemblance avec les cours de

19. Cf. Godefroy (1880-1902) et l'édition de La Curne de Sainte-Palaye (1875-1882). Voir note 24, infra. 
littérature française, les romanistes durent imposer de nouveaux thèmes, en fonction des œuvres du moyen-âge. Ce fut un des usages que reçut «l'épopée».

\section{Un nouvel objet : l'épopée :}

Le premier professeur à entretenir son auditoire d'épopée fut Delavigne (Toulouse, 1859) : " (le professeur) exposera l'histoire de la littérature française au moyen-âge. Il étudiera d'abord les principaux monuments de l'histoire et de l'épopée au XIII ${ }^{\mathrm{e}}$ et au XIV siècles ». En 1865, de nouveau : "le professeur étudiera, dans leur apparition successive, les œuvres caractéristiques de l'esprit français, pendant la durée du moyen-âge. Il analysera tour à tour les épopées chevaleresques et allégoriques, les mémoires historiques (Villehardouin, Joinville, Froissard) ; et dans le second semestre, il s'occupera surtout des origines et des progrès du théâtre, pendant le $x^{e}$ et le $\mathrm{XVI}^{\mathrm{e}}$ siècles ".

Le projet de Delavigne s'inscrit dans la conception la plus traditionnelle de l'enseignement des lettres médiévales : suivre les progrès d'une littérature qui passe de la fabulation épique anonyme à une littérature profane - le théâtre - après un détour par l'histoire. La même démarche se lit en filigrane dans son cours de 1873 : «Le professeur fera l'histoire des lettres françaises au moyen-âge. Il s'occupera d'abord de l'épopée et commencera par l'étude du cycle Carlovingien, et particulièrement de la Chanson de Roland».

Comment expliquer cet intérêt du doyen de la faculté des lettres de Toulouse pour la littérature médiévale? Probablement, par le souci de se poser en prétendant à la succession de Paulin Paris au Collège de France. On sait que Gaston Paris recevra la chaire de son père définitivement en 1872, profitant non seulement d'un népotisme bien compris mais surtout de sa conformité aux nouvelles exigences : maîtriser l'explication linguistique dans l'analyse philologique des textes. Entre Delavigne et G. Paris, le choix opéré n'est pas celui de la capitale contre la province, ou d'une génération (Delavigne est né en 1817, G. Paris en 1839), mais celui qui sépare encore aujourd'hui le littéraire du philologue. 
Lorsque Delavigne annonce son dernier cours, d'autres ont repris ou vont reprendre le mot "épopée » pour explorer d'autres domaines :

- Baret (Clermont-Ferrand, 1866), dans la chaire de « Littérature Étrangère ", traite de "l'épopée chevaleresque, particulièrement en Espagne et en Italie »;

- Courdaveaux (Douai, 1871), en «Littérature Ancicnne ", fait un cours sur «les épopées indiennes»;

- Duchesne (Rennes, 1872) sur l' «Histoire de la poésie épique en France ", en « Littérature Française »;

- Benoist (Aix, 1873), en "Littérature Étrangère »: «La poésie lyrique et la poésie épique en Allemagne au moyen-âge " ;

- Petit de Julleville (Dijon, 1874) : «De l'épopée française au moyen-âge » en "Littérature Française » - ce dernier cours peut être suscité par l'annonce d'une chaire de littérature médiévale française à la Sorbonne ${ }^{20}$;

- Hallberg (Dijon, 1876), en « Littérature Étrangère », se consacre à "l'épopée chrétienne dans les littératures étrangères ».

Passé cette date, et à deux exceptions près ${ }^{21}$, tous les cours sur l'épopée concerneront exclusivement la littérature épique du moyenâge en France : la présence, dans les programmes de la licence et de l'agrégation de textes en ancien français, spécialement La Chanson de Roland ${ }^{22}$, y contribue puissamment.

A contrario, il n'existe jamais, dans aucune chaire, de cours sur l'épopée grecque ou latine. L'épopée est réservée aux médiévistes (au

20. Petit de Julleville l'obtiendra d'ailleurs, après la disparition de Darmesteter en 1888.

21. Joret (Aix, 1880) : «Histoire de la poésie épique et lyrique au moyen-âge, principalement en Allemagne et en Italie », et Castets (Montpellier, 1884-85) : «L'épopée italienne au moyen-âge ».

22. Il faut souligner combien Roncevaux, cette histoire de bons et de méchants, avec l'argument d'une défaite qu'efface une victoire, pouvait plaire à des républicains imprégnés par le thème de la Revanche. Déjà, en 1871, Charles Lénient n'avait pas craint d'intituler son cours de la Sorbonne : « La poésie patriotique en France au moyen-âge ", sans omettre de signaler l'actualité de son sujet. 
même titre que les "mystères" qui constitueront le thème de prédilection de Petit de Juleville), mais les cours sur ce sujet restent en nombre limité :

- Darmesteter (Paris, 1877 et 1881); Clédat (Lyon, 1879); Aubertin (Dijon, 1881); Thomas (Toulouse, 1883 et 1884); Ducros (Poitiers, 1889); Stapfer (Bordeaux, 1892); Jeanroy (Toulouse, 1894 et 1895).

Qu'indique ce tableau? La coïncidence chronologique de la première génération des romanistes - Darmesteter et Clédat - et des derniers rhéteurs formés par Villemain et Saint-Marc-Girardin, comme Aubertin, auxquels succède une nouvelle génération illustrée par Jeanroy. Désormais, "épopée " est associée à littérature française du moyen-âge et au nouveau paradigme de la romanistique, exclusivement. À défaut d'imposer l'enseignement de la syntaxe française ou de la phonétique dans les facultés de lettres, les comparatistes ont dû se revendiquer de formes originales de la littérature médiévale pour s'imposer face aux détenteurs de chaires de "Littérature Française » qui refusent d'étendre à la « Langue " la compétence de leur magistère.

En somme... :

S'il a fallu l'orgueil blessé d'une nation et la nécessaire refonte de tout l'enseignement supérieur pour que l'AF puisse figurer dans les programmes de la licence et de l'agrégation, les exigences d'un concours qui recrutait des enseignants pour des lycées où l'AF était absent contribuèrent à minorer une discipline dominée par les études classiques et le prestige de la littérature française moderne. Afin d'obtenir leur reconnaissance, les médiévistes furent obligés de composer avec la routine des facultés de lettres en privilégiant la littérature contre la langue ${ }^{23}$ et en confortant, par leurs enseignements, la doxa républicaine.

23. A. Darmesteter, pour être docteur, dut soutenir sa thèse mineure en littérature sur le Floovent. Il lui fut bien sûr reproché par un des membres du jury de n'avoir prévu aucune appréciation esthétique et de s'en être tenu à une analyse strictement philologique. 
D'un Roland transformé en Père-la-Revanche à la proclamation de l'unité linguistique gallo-romane ou au culte du francien, le romanisme en France, pour répondre aux attentes de ses fondateurs (assurer aux textes médiévaux, domaine d'élection de la philologie allemande, une exploitation nationale de qualité), devait se plier aux exigences de l'enseignement supérieur. Et si les romanistes échouèrent dans l'imposition de la phonétique, de la dialectologie et de la grammaire historique et comparée à l'intérieur des facultés de lettres, au moins justifièrent-ils la patrie. Une et vénérable nation qui se perpétuait de l'ancien français au français contemporain ${ }^{24}$.

Gabriel BERGounioux.

24. Bibliographie : Gérald Antoine et Robert Martin (dir.), Histoire de la langue française 1880-1914, Paris, 1985 - Gabriel Bergounioux, L'enseignement de la linguistique et de la philologie en France au XIX siecle d'après les affiches de cours des facultés de lettres, dans Archives et documents de la SHESL, seconde série, ${ }^{\circ} 2,1990$; Gabriel Bergounioux, Linguistique et variation : reperes historiques, dans Gadet, voir infra Linguistique et philologie dans les facultés françaises (1850-1900), dans Actes du Colloque tenu pour le Centenaire de l'E.L.C.F. (Genève) [à paraître] - Anatole Boucherie, Procès-verbal de la séance du 17 aril 1869, dans Bull. de la Société pour l'Étude des Langues Romanes, A. Franck, 1870 - Bernard Cerquiglini, La naissance du français, Paris, 1991 - Jean-Claude Chevalier, Les grammaires et l'histoire de la langue, dans G. Antoine et R. Martin (1985) p. 577-600 - Léon Clédat, Grammaire élémentaire de la vieille langue française, Paris, 1885 - Antoine Compagnon, La Troisième République des Lettres, de Flaubert à Proust, Paris, 1983 - Arsène Darmesteter, Cours de grammaire historique de la langue française (I-IV), Paris, 1891-189? -Françoise Gadet (dir.), "La variation " Langages, Paris, 1992 — Frédéric Godefroy, Dictionnaire de l'ancienne langue française et de tous ses dialectes, I-IX, 1880-1902 - Adolphe Hatzfeld et Arsène Darmesteter, Dictionnaire Général de la Langue Française, Paris, 1890 - dans Gaston Paris, «Les parlers de France ", dans Bull. de la Société des Parlers de France, I, 1 (1893), p. 1-19 - Antoine Prost, Histoire de l'enseignement en France (1800-1967), Paris, 1970. 